\title{
Using Single Sign-On Authentication with Multiple Open OnDemand Accounts: A Solution for HPC Hosted Courses
}

\author{
Ping Luo \\ Yale Center for Research Computing, \\ Yale University \\ New Haven, CT, USA \\ ping.luo@yale.edu \\ Kaylea Nelson \\ Yale Center for Research Computing, \\ Yale University \\ New Haven, CT, USA \\ kaylea.nelson@yale.edu
}

\author{
Benjamin Evans \\ Yale Center for Research Computing, \\ Yale University \\ New Haven, CT, USA \\ b.evans@yale.edu
}

Thomas J. Langford

Yale Center for Research Computing, Yale University

New Haven, CT, USA

thomas.langford@yale.edu

\author{
Andrew Sherman \\ Yale Center for Research Computing, \\ Yale University \\ New Haven, CT, USA \\ andrew.sherman@yale.edu
}

\author{
Tyler Trafford \\ Yale Center for Research Computing, \\ Yale University \\ New Haven, CT, USA \\ tyler.trafford@yale.edu
}

Jay Kubeck

Yale Center for Research Computing,

Yale University

New Haven, CT, USA

jay.kubeck@yale.edu

\begin{abstract}
Open OnDemand (OOD) greatly lowers the barrier to entry to high performance computing (HPC) resources and facilitates usage for new and experienced users. Moreover, using OOD for courses with computational components enables lecturers and teaching assistants to focus on the course instead of on-boarding students. To these ends the Yale Center for Research Computing (YCRC) adopted OOD to make its advanced cyberinfrastructure more accessible to researchers and students across the university. While the use of single sign-on authentication in OOD makes HPC clusters easier to access, as implemented this authentication method limits users to a single HPC account per university-provided identity. The YCRC provides separate and temporary course-specific accounts to isolate course usage, which presented a challenge for supporting users with pre-existing research allocations or who are participating in multiple courses. In this paper, we present an easy to implement and maintain solution that separates traffic for each course and maps a single user identity to multiple HPC accounts. This solution can be used for other situations other than courses; wherever one single sign-on identity should access multiple HPC accounts.
\end{abstract}

\section{KEYWORDS}

Open OnDemand, web portal, HPC, clusters, cyberinfrastructure, single sign-on, CAS, Apache, virtual host, support for classes, courses, academic support, students, education

\section{(c) (7) (8)}

This work is licensed under a Creative Commons Attribution-NonCommercial International 4.0 License.

PEARC '21, fuly 18-22, 2021, Boston, MA, USA

(c) 2021 Copyright held by the owner/author(s).

ACM ISBN 978-1-4503-8292-2/21/07.

https://doi.org/10.1145/3437359.3465575

\section{ACM Reference Format:}

Ping Luo, Benjamin Evans, Tyler Trafford, Kaylea Nelson, Thomas J. Langford, Jay Kubeck, and Andrew Sherman. 2021. Using Single Sign-On Authentication with Multiple Open OnDemand Accounts: A Solution for HPC Hosted Courses. In Practice and Experience in Advanced Research Computing (PEARC '21), fuly 18-22, 2021, Boston, MA, USA. ACM, New York, NY, USA, 6 pages. https://doi.org/10.1145/3437359.3465575

\section{INTRODUCTION}

The Yale Center for Research Computing (YCRC) supports research computation at Yale by providing high performance computing (HPC) resources and expertise. The YCRC operates four HPC clusters comprising about 35,000 cores, 100 s of GPUs and over $10 \mathrm{~PB}$ of GPFS cluster storage that are used by thousands of researchers in hundreds of different groups across Yale University and its professional schools. The YCRC adopted Open OnDemand (OOD) [6] to forward its mission of making advanced cyberinfrastructure more accessible to the research and academic communities at Yale. We chose to set up the YCRC HPC portal using OOD because it is a general-purpose portal created for HPC centers [6] and is easy to use. Before OOD was released, many web portals from academia have been developed for special uses. For example, Galaxy [5] is used by researchers for biology-specific workflows and Globus Online [4] is used for data transfer only. None of these portals alone are able to address the general needs of an HPC center and its users as OOD does. OOD has been deployed by more and more universities and organizations [3] and the number of sites continues to grow.

Open OnDemand has proven invaluable for lowering the barrier to entry into HPC for users at Yale, particularly those with minimal technical backgrounds. It is regularly employed by the YCRC research support staff when on-boarding new users, but has been adopted by advanced users as well. Regardless of skill level, OOD reduces friction in many common pain points. All users of 
YCRC supported OOD portals can authenticate easily with university identities provided via the Central Authentication Service (CAS) [1], Yale's single sign-on (SSO) authentication service for all faculty, staff and students. All users can easily log in with their university identity, transfer files, start a remote desktop session, and interact with familiar applications like Jupyter and RStudio.

In addition to enthusiastic adoption by the research computing community, OOD has enabled HPC access to academic courses at Yale. Historically, YCRC has provided HPC resources to Yale courses with an appropriate computational component in the form of temporary (i.e. deleted after the semester ends) accounts for the faculty, students, and teaching assistants, dedicated compute resources, application support and cluster storage. The introduction of OOD has made this service highly appealing to faculty. Lecturers find they spend less in-class time teaching basic HPC and Linux skills as many students arrive comfortable with a web interface. The lecturer and teaching assistants (TAs) save time by providing and supporting a universal set of instructions when the HPC interface is a web portal; which operating system the students run becomes almost inconsequential when they all use a web browser for access. Moreover, TAs can focus on supporting course subject matter instead of troubleshooting issues with SSH keys and fielding questions on command line syntax. For example, the OOD Jupyter application has been particularly successful in courses. All involved have found this replacement for staking out ports and ssh tunnels a welcome change.

These course accounts present new administrative and technical challenges, though. A student's temporary account name takes the form of courseid_username to avoid identity namespace collisions with research accounts. For cases such as this where the CAS identity does not match the HPC account name, the YCRC uses a "user mapping script" to link the two identities. This allows users to authenticate via CAS but access their temporary course accounts. However, OOD can only be configured to log each authenticated identity to a single local account, so students enrolled in multiple courses taking advantage of HPC resources or who are also engaged in research were not able to use OOD with all of their accounts.

Here we present a solution for handling multiple accounts while still using single sign-on authentication for Open OnDemand. We use Apache name-based virtual hosts to separate the traffic into standard access and logins for individual courses. The OOD servers then use a customized user mapping script to log the user into the correct target HPC account. This allows a user to use the regular cluster OOD URL to access their research account and customized course OOD URLs to access any number of course accounts. This solution has the added benefit of letting us customize the portal for each course. In the following sections, we describe the YCRC OOD environments, our customized user mapping solution, and how that solution improves the experience for courses and for users with multiple accounts on our HPC clusters.

\section{OPEN ONDEMAND AT THE YCRC}

The YCRC maintains and supports four HPC clusters: two generalpurpose clusters, Grace and Farnam, and two smaller special use clusters, Ruddle and Milgram. The majority of courses are allocated resources on the Grace and Farnam clusters. Each of the clusters has a separate primary shared filesystem and separately scheduled down times for updates and maintenance. We dedicate a node from each cluster as an OOD server that is reconfigured and provisioned during cluster maintenance using Ansible. Each OOD server has 16 CPU Cores and $128 \mathrm{GiB}$ of RAM. We also have a virtualized OOD instance, where we test new OOD releases and develop new apps and features.

Generally speaking, an OOD server consists of two parts: the Apache HTTP server frontend, which runs as the Apache user and serves as a proxy server, and the Per-User Nginx (PUN) backends, each of which runs as an authenticated user. User authentication is configured at the Apache frontend.

Our OOD servers all use CAS as the authentication provider. CAS is a single sign-on protocol which was originally designed and developed at Yale in 2000 to 2002. It was released to the public domain in 2004 and has been adopted by many universities. The Yale CAS server, maintained by the Yale IT department, authenticates via a centrally managed university identity, called a NetID, and its associated password. Aside from the benefit of single sign-on, using CAS for the YCRC OOD services also free us from the tasks of identify management.

\section{AUTHENTICATION AND USER MAPPING}

OOD provides two scripts that allow administrators to choose how to map authenticated username to local accounts. One uses regular expressions for the mapping and the other uses a lookup file.

The ood_auth_map.regex script uses a regular expression (regex) to map authenticated users to local users and is the default user mapping method. It takes a username as an input and prints a regex transformed name to the standard output. The default regex is $+^{\wedge}(.+) \$+$, which echoes the input to the output unchanged. The regex can be changed with the option --regex=REGEX. For example,

ood_auth_map.regex regex='^( $\left.\backslash w^{+}\right) @ y a l e . e d u$ alice@yale.edu will only print the mapped name before + ' $Q$ ' + if the domain matches and return true. If the domain does not match it will print an empty string and return false, resulting in a failed login.

The ood_auth_map.mapfile script allows administrators to use an explicit authenticated username to local cluster username lookup table. It takes an authenticated username as an input and prints the mapped name to the standard output by scanning a lookup file (mapfile). Each line in the mapfile is in the space-delimited two-column format:

$$
\text { "authenticated_user" local_cluster_account }
$$

The ood_auth_map.mapfile script prints the second column from the same line where it finds the first exact match for the provided input username in the first column. If no match is found, the script prints an empty string and exits with false, resulting in a failed login. By default the file /etc/grid-security/grid-mapfile is used, unless a different one is specified with $-f / p a t h / t o / m a p f i l e$.

To use CAS authentication, we register the URL for OOD with the CAS server, and configure the CAS login URL in the OOD Apache frontend. Once configured, a user who visits the OOD URL will be redirected to the CAS server to authenticate with their username (called a NetID), password, and a second factor provided by Duo Multifactor Authentication. The CAS server sends back a signed 
ticket with the user's NetID if the user successfully authenticates. The Apache frontend stores this NetID in the environment variable REMOTE_USER, which is passed to the user mapping script as the input username [2]. The local cluster username returned by the mapping script is then used by OOD to start the PUN instance for the authenticated user.

\section{HANDLING MULTIPLE HPC ACCOUNTS FOR A SINGLE CAS IDENTITY}

While the OOD provided user mapping script is an elegant solution to linking single sign-on identities and HPC accounts, the one-toone mapping is a limitation for users with multiple HPC accounts. A single user could have an HPC account for research and multiple course accounts, all of which they would like to use with OOD. To address this challenge, we designed and implemented a solution which makes use of Apache name-based virtual hosts to separate research and course account traffic, and a customized user mapping script to handle the identity mapping accordingly.

\subsection{Apache Name-based Virtual Host}

Apache httpd supports name-based virtual hosts, which is ideal for implementing multiple OOD access points for different courses on the same server. This technology allows different virtual hosts, each with its own DNS name, to share the same IP address on a single Apache server. Herein we use the term "portal" to delineate the corresponding virtual host presented to a user when they access our server with a specific OOD custom URL. Using name-based virtual hosts, we are able to create a general portal and multiple course portals on a single OOD server. Unless restrictions require us to apply access controls, the general portal is opened to all users with a research account on the cluster. When a user CAS authenticates to the general portal with their NetID, the user enter the OOD dashboard as their research HPC account (where their username is their NetID). We DNS register names for course portals following the convention courseid.ycrc.yale.edu to give memorable URLs to each virtual host. When a student accesses their course portal, the student still CAS authenticates with their normal credentials. Once logged in, the student will enter an OOD session as their course HPC account (with the username following our convention for temporary course accounts of courseid_NetID). Each course portal is only open to the students who have enrolled in that course. Any access attempts to the course portal from a non-course user are denied.

\subsection{Hybrid User Mapping Scheme}

Apache name-based virtual hosts separate traffics from different types of users through different URLs. This opens us to the possibility to map the users differently depending on the URL used to access the server. With that in mind, we need a mapping mechanism that can:

(1) Map user NetID to research accounts for general portal users.

(2) Map user NetID to their corresponding course account for course portal users.

(3) Only allow enrolled students from each course access to the corresponding portal.
(4) Allow administrators to become other users on the cluster to help troubleshoot issues.

To achieve these objectives, we designed and implemented a hybrid user mapping scheme that combines the regex user mapping method with the lookup file user mapping method. The pseudo code of the new mapping scheme is:

(1) If the default mapfile exists, then scan username in the default mapfile

(a) If found, print the local_cluster_user from the first match in the mapfile and return true.

(2) If a customized mapfile is provided, then scan username in the mapfile.

(a) If found, print the local_cluster_user from the first match in the mapfile and return true.

(b) Else, print a blank line and return false.

(3) Else parse username through regex.

(a) If succeed, print the parsed name and return true.

(b) Else, print a blank line and return false.

(4) For all other cases, print a blank line and return false.

\subsection{How It Works}

Our solution integrates Apache virtual hosts with our customized user mapping script called user_auth_map.ycrc, which is based on the pseudo code described above. We will describe an example (also shown in Fig. 1) to illustrate how our approach works. The example is a simple demonstration of the workflow, but is an accurate representation of our real use cases.

First let us examine our virtual host setups. Suppose we are offering two courses on the cluster. We need at least three virtual hosts on the Apache OOD proxy server. One is the general portal, which is open to all users with research accounts, and one course portal for each course. The user mapping command for the general portal does not take a mapfile as an argument. The user mapping commands of the course portals, on the other hand, each have their own customized mapfile that contains course users.

In this example, there are two users with different account setups. User Alice's NetID is alice123 and she has a research account and is enrolled in course A. Her research account shares an account name with the user's NetID and her course account is courseA_alice123. User Bob's NetID is bob123 and he is taking course A and course B, but has no research account. He, therefore, has two course accounts: courseA_bob123 and courseB_bob123.

Now, let us see what will happen when these users access the three portals. Let us further assume both have already passed the CAS authentication and, therefore, their authentication sessions are waiting to be mapped by the mapping commands of the three portals to the correct target HPC accounts.

- general portal

- For Alice, her NetID will be mapped to alice123. Since the research account alice 123 exists on the cluster, a PUN owned by alice123 will be started on the cluster and Alice will be logged into the general portal successfully. 


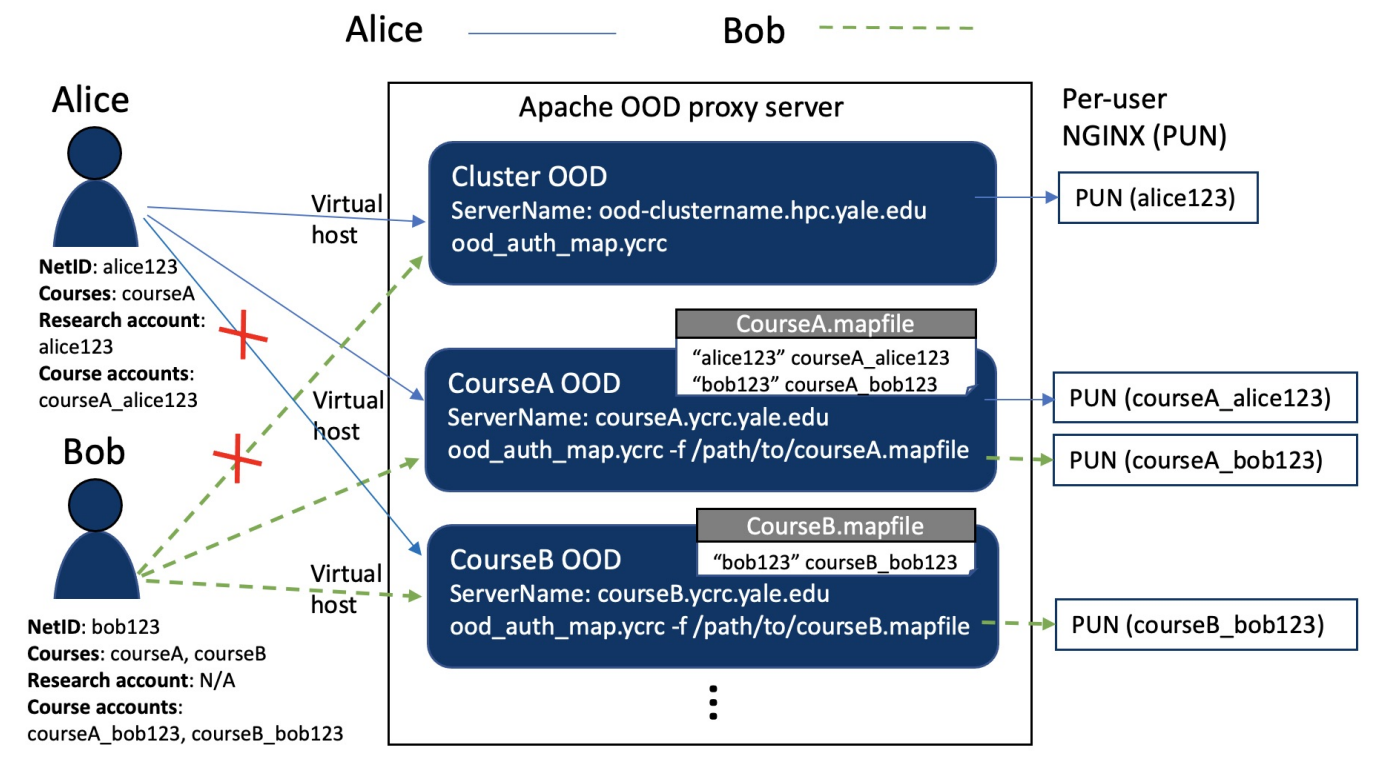

Figure 1: Workflow for mapping users in different portals on the same OOD server

- For Bob, his NetID will be mapped to bob123. Since there is no such a research HPC account on the cluster, it will fail when OOD tries to start a PUN for bob123. Bob will get an error stating that user bob123 cannot be found. Consequently, Bob will not be able to $\log$ in to the general portal.

- courseA portal

- For Alice, her NetID alice123 will be mapped to her course account, which is courseA_alice123 as specified in courseA.mapfile. Since courseA_alice123 exists on the cluster, Alice will be logged into courseA.ycrc.yale.edu as courseA_alice123.

- For Bob, his NetID bob123 will be mapped to his course account courseA_bob123, as specified in courseA.mapfile. Since the account courseA_bob123 also exists on the cluster, Bob will be logged in as courseA_bob123.

- courseB portal

- Alice is not a student in course B. So, her NetID does not appear in mapfile for course B. Therefore, the user mapping command cannot find a match of alice 123 and the login to courseB.ycrc.yale.edu will fail for Alice.

- Bob, on the other hand, will be successfully logged into courseB.ycrc.yale.edu as courseB_bob123.

Finally, an admin can impersonate a user on the cluster for troubleshooting. For a course portal, the admin can add an entry to the course mapfile in the format:

$$
\text { “'admin_NetID', course_account }
$$

When the admin authenticates to CAS with their NetID, they will enter an OOD session as the specified course HPC account. For the general portal, the admin needs to use /etc/ood/config/map_file to map their NetID to the user's research account.

\subsection{Additional Features}

4.4.1 Automap. In addition to mapping through regex or mapfile, ood_auth_user.ycrc supports a completely new mapping method called automap. Automap uses a string as the template and replaces a sub-string with the authenticated username. The template string is taken from the input specified by the option '-a' or '--automap'. The template string must contain a percent sign (\%) as the sub-string to be replaced. As an example,

$$
\text { ood_auth_user.ycrc -a courseA_\% alice123 }
$$

will print courseA_alice123. This is very useful for our specific use case since our course accounts are NetIDs prefixed by a course name.

4.4.2 Customized Portals. Creating a separate Apache virtual host for each group of accounts allows for further customization of OOD than is typically possible when providing access to a general purpose HPC cluster. In the case of the YCRC, this has allowed for simplification and streamlining of the student experience. Since we DNS register each virtual host, each course has a relevant address that students can more easily remember. We can also change OOD app availability on a per-course basis. For example, one course may want students to use the same Python environment to work in Jupyter notebooks, and at the same time to not be distracted by other interactive applications. To do this we can hard-code and simplify the more flexible Jupyter form we provide to the general research community, and remove references to other applications such as MATLAB and RStudio.

\subsection{Virtual Machine vs Apache Virtual Host}

An alternative approach we considered was setting up a virtual machine (VM) for each course to run OOD. VMs would allow customization of the OOD dashboard with individualized banner and 
MOTD, since each course would have its own dedicated OOD server. However, the resource requirements and the administration costs are much higher with that approach. Virtual hosts, on the other hand, are very lightweight. There is only one server shared by many courses. However, this means all the courses share the same dashboard. After comparing the pros and cons, we chose virtual hosts for our solution.

\subsection{Use Cases}

We have set up OOD portals to nine courses on our two major clusters, serving 530 students. Table 1 shows the course names and number of students in each course. As the semester progresses, we expect additional courses will request their own course portals.

\begin{tabular}{|l|c|c|}
\hline Cluster Name & Course Name & Number of Students \\
\hline \multirow{4}{*}{ Grace } & CPSC 424 & 45 \\
\cline { 2 - 3 } & S\&DS 123 & 199 \\
\cline { 2 - 3 } & SOCY 163 & 12 \\
\cline { 2 - 3 } & CHEM 513 & 18 \\
\cline { 2 - 3 } Farnam & S\&DS 317 & 73 \\
\hline \multirow{5}{*}{} & ANTH 395 & 8 \\
\cline { 2 - 3 } & BENG 469 & 17 \\
\cline { 2 - 3 } & CPSC 452 & 125 \\
\cline { 2 - 3 } & GENE 760 & 33 \\
\hline
\end{tabular}

Table 1: Courses on Grace and Farnam with an OOD portal

\subsection{Security}

Our solution relies on mapfiles extensively. Since a mapfile could allow a user to access the portal as any other user, the files must be carefully protected. As such, all the mapfiles are owned by root and, while they must be world readable, they are only writable by root. In the future, the course mapfiles could potentially be made accessible to TAs for individual course portals.

\section{CONCLUSION}

In this paper, we present a simple solution for enabling users with multiple accounts to access the Open OnDemand server configured with CAS. This solution uses Apache virtual hosts and our customized user mapping script to create an extra mapping layer that allows more flexible routing of authentication to HPC accounts and customization of separate virtual hosts.

The YCRC has leveraged this approach to make hosting courses more streamlined. Students are given separate storage and scheduler accounts for coursework that do not interfere or commingle with their research work. Moreover, by providing isolated course accounts, we can provide these accounts on a temporary basis and purge the whole course from the cluster at the end of course duration, leaving a clean slate for the next offering. This could also be applied to other use cases, e.g. project-based allocations where users or administrators want harder separation between allocations. Moreover, administrators can edit the mapfile by hand to impersonate any user on the system, which has proven immensely useful in troubleshooting.
Open OnDemand has significantly increased the accessibility of HPC resources to both researchers and students at Yale. The approach described in this paper enables users who are both researchers and students (or students in multiple courses) to continue to use SSO authentication while accessing their multiple HPC accounts. Apache name-based virtual hosts with a customized user mapping script provide an easy to implement and maintain solution that can be hosted on a single Open OnDemand server. Our solution also allows us to provide courses with a portal customized for its curriculum and that reduces on-boarding time. This solution could be generalized to any situation where individual users want to use Open OnDemand with SSO authentication to access multiple accounts. Our customized user mapping script for Open OnDemand is available on GitHub at https://github.com/ycrc/ood-user-mapping.

\section{FUTURE WORK}

While this solution has been effective in addressing our primary issue of accessing multiple HPC accounts with one SSO service, we see the potential for a few improvements and additional applications. One additional application would be enabling TAs to more efficiently assist with student HPC accounts. A TA who works with a course may need access to a student's home directory to either help the student with questions related to the coursework or check the student's assignments. Currently, we provide this access to TAs by giving their HPC accounts read and executable permissions to the student's directories using access control lists (ACLs). However, this approach is often insufficient for more complicated troubleshooting that could be more easily addressed if the TA could $\log$ in as the student HPC accounts. Using the OOD mapfile for the course, system administrators can map the TA to the student and then the TA can start an OOD session in the student's account. We would like to explore to implement a set of tools that would allow the TA to manage the mapfile for their associated course so that they could $\log$ in as any of the student HPC accounts.

\section{ACKNOWLEDGMENTS}

We thank Eric Franz and Trey Dockendorf from Ohio Supercomputing Center for feedback on our early ideas for resolving the issue faced by our users with multiple HPC accounts when accessing OOD. The complexity of those ideas has inspired us to seek this simple but effective solution. We also thank Rob Bjornson, Michael Strickler and the rest of the YCRC for their assistance with testing our Open OnDemand instances and solution. We appreciate the participation, thoughtful feedback and support from our users, the researchers, faculty and students at Yale University. Their computational needs and interest inspired this work. This work used resources hosted and provided by the Yale Center for Research Computing.

\section{REFERENCES}

[1] [n.d.]. CAS - Central Authentication Service | Yale API. https://developers.yale. edu/cas-central-authentication-service. Accessed: 2021-03-08.

[2] [n.d.]. OOD User Guide - Setup User Mapping. https://osc.github.io/ooddocumentation/latest/authentication/overview/map-user.html. Accessed: 202103-08.

[3] Alan Chalker, Eric Franz, Morgan Rodgers, Trey Dockendorf, Doug Johnson, Doris Sajdak, Joseph P. White, Benjamin D. Plessinger, Mohammad Zia, Steven M. Gallo, Robert E. Settlage, and David E. Hudak. 2020. Open OnDemand: State of the 
platform, project, and the future. Concurrency and Computation Practice and Experience (2020), e6114. https://doi.org/10.1002/cpe.6114

[4] Ian Foster. 2011. Globus Online: Accelerating and democratizing science through cloud-based services. IEEE Internet Computing 15, 3 (2011), 70-73. https://doi.org/ 10.1109/MIC.2011.64

[5] Belinda Giardine, Cathy Riemer, Ross C Hardison, Richard Burhans, Laura Elnitski, Prachi Shah, Yi Zhang, Daniel Blankenberg, Istvan Albert, James Taylor, Webb
Miller, W James Kent, and Anton Nekrutenko. 2005. Galaxy: a platform for interactive large-scale genome analysis. Genome Research 15, 10 (2005), 1451. https://doi.org/10.1101/gr.4086505

[6] Dave Hudak, Doug Johnson, Alan Chalker, Jeremy Nicklas, Eric Franz, Trey Dockendorf, and Brian L. McMichael. 2018. Open OnDemand: A web-based client portal for HPC centers. Journal of Open Source Software 3, 25 (2018), 622. https://doi.org/10.21105/joss.00622 